Gefässchirurgie 2020 · 25:385-386 https://doi.org/10.1007/s00772-020-00694-5 Online publiziert: 15 . September 2020 (c) Springer Medizin Verlag GmbH, ein Teil von Springer Nature 2020

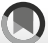

K.-H. Rendl

Salzburg, Österreich

\title{
Peter C. Maurer - vidit, venit, vicit
}

\section{Zur Gründung der Zeitschrift Gefässchirurgie vor 25 Jahren}

Den in deutscher Sprache publizierenden Gefäßchirurgen standen in den achtziger Jahren mit der von Peter Maurer bereits 1978 ins Leben gerufenen und bis 1994 erschienenen Zeitschrift „Angio" und der schweizerischen „Vasa“ "zwei Publikationsorgane zur Verfügung, die beide den nationalen Fachgesellschaften als jeweils „offizielles Organ“dienten. Ansonsten wurde mit deutlicher Zurückhaltung in der „Deutschen Zeitschrift für Herz-, Thorax- und Gefäßchirurgie“, in den Zeitschriften zur Allgemeinchirurgie oder in englischsprachigen Gefäßjournalen veröffentlicht.

Nach Ende der Ära "Angio - Zeitschrift für Gefäßchirurgie, Angiologie, Angioradiologie“, der ihre Gründer damals schon in weiser Voraussicht einen breitgefassten Untertitel gegeben hatten, und da die „VASA“ eher die Gefäßmedizin repräsentierte, standen die rasch gewachsenen gefäßchirurgischen Fachgesellschaften 1994 ohne eigenes Publikationsorgan da. Dieses Vakuum musste ehest beseitigt werden. Wie mehrfach vorher sprang Prof. Dr. Peter C. Maurer ein, den ohnehin immer ein feines Gespür für politische Probleme der Gefäßchirurgie und ihrer Zukunft auszeichnete. Auf seine Initiative wurde erstmals 1994 mit Vertretern der gefäßchirurgischen Gesellschaften Deutschlands, Österreichs und der Schweiz über Grenzen hinweg der Gedanke an die Gründung einergemeinsamen neuen Gefäßzeitschrift in Zusammenarbeit mit einem international tätigen und bekannten Verlag andiskutiert. Von Beginn an gefordert waren ein funktionierendes Peer-ReviewSystem und die Ausdehnung der Inhalte über die Gefäßchirurgie hinaus zu allen themenverwandten Fragen.
Diese ersten von Maurer als "Chefgründer" gesuchten Gespräche verursachten bei allen Angesprochenen ein Kooperationsfeuer. Maurer gelang es, in kürzester Zeit eine Ärztecrew, vor Ort unterstützten ihn H.M. Becker und J. Allenberg, und Vertreter des SpringerVerlags um sich zu scharen, die mit ihm und dem ersten Schriftleiter Hans Martin Becker dieses Projekt so intensiv vorantrieben, dass bereits 1996 die ersten vier Hefte erscheinen konnten. In der Folge wurden von 1997 bis 2020 2029 Publikationen in 157 Ausgaben bzw. 24 Bänden veröffentlicht [1].

》) Von 1997 bis 2020 wurden in 157 Ausgaben 2029 Publikationen veröffentlicht

Die „Gefässchirurgie - Zeitschrift für Vaskuläre und Endovaskuläre Medizin", wie sie inzwischen heißt, existiert nun seit 25 Jahren. Heute liest sie wahrscheinlich fast jeder deutschsprachige Gefäßchirurg regelmäßig, weil der strukturierte Mix aus Wissenschaftlichkeit, praktischen Hinweisen und Fortbildung bei gleichzeitiger Information der Fachgesellschaften anspricht.

Herausgeberkollegium und Schriftleiter haben gewechselt, der Inhalt wurde geschärft, es wurden Rubrikenherausgeber benannt, der wissenschaftliche Beirat gibt das aktuelle „Who is Who" der Gefäßchirurgie wieder. Die Zahl der jährlichen Hefte stieg von vier auf acht, die Auflage pro Heft um stolze $250 \%$, sodass auch die Budgets der Gesellschaften nicht mehr allzu sehr belastet werden.

Kein Erfolg ohne etwas Salz in der Suppe, wie es Herausgeber und Schriftleiter bezieht G. Matius; Zitiert nach Plutarch (?). 


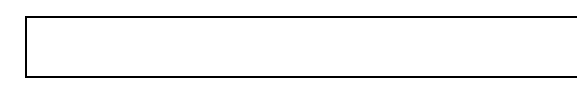

in ihren Beiträgen wiederholt angesprochen haben und auch in der Festschrift zum Jubiläumsheft diskutiert wird. Man darf hoffen, dass auch in deutschsprachigen Landen das Englische als führende medizinische Wissenschaftssprache vermehrt geübt und verstanden wird. Um eine sprachliche Internationalisierung, wie sie schon Eckstein favorisiert hat, wird man wohl auf Dauer nicht herumkommen, wenn man eine Weiterentwicklung der Zeitschrift anstrebt.

Lieber Peter, Du kannst, denke ich, mit Stolz auf Deine Gründung schauen. Dir allein gebührt das Primat, das Projekt angestoßen zu haben. Wir, die wir mitarbeiten durften, danken Dir dafür und denken, dass diese Zeitschrift für alle drei Fachgesellschaften gleichermaßen von hervorragender Bedeutung war und ist. Sie dient nicht nur als Sprachrohr, nein, sie ist auch für die Identität der deutschsprachigen Gefäßchirurgie von außerordentlicher vielleicht in ihrer Tragweite nicht immer erkannten Wichtigkeit.

Ihr

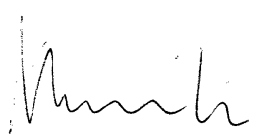

Univ. Doz. Dr. Karl-Hans Rendl

\section{Korrespondenzadresse}

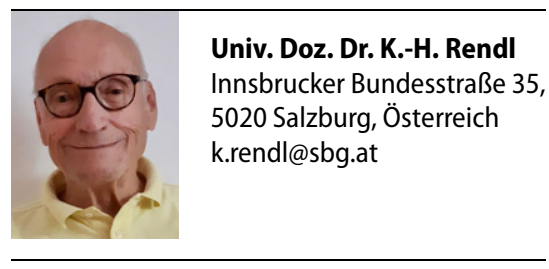

Interessenkonflikt. K.-H. Rendl gibt an, dass kein Interessenkonflikt besteht.

\section{Literatur}

1. Eckstein HH, Söllner $H$, Kühnl A (2015) Die Zeitschrift Gefässchirurgie von 1996 bis 2015 Bibliometrische Analyse und inhaltliche Perspektiven. Gefässchirurgie 20:304-312

\section{Veranstaltungstipp}

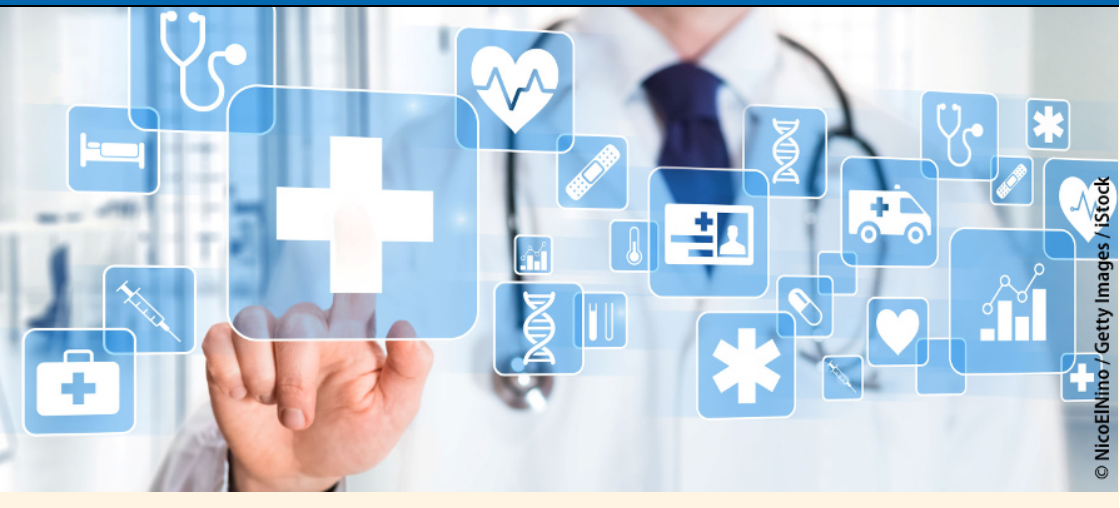

\section{DGIV-Bundeskongress, 29. Oktober $\mathbf{2 0 2 0}$}

Überwindung der Sektorengrenzen - Erkenntnisse aus der CORONA-Krise

Die Deutsche Gesellschaft für Integrierte Versorgung e.V. ist ein deutschlandweit agierender Verein mit der Zielsetzung, die Integrierte Versorgung in der medizinischen, pflegerischen und sozialen Betreuung als Regelfall durchzusetzen und die dazu erforderliche Beseitigung der noch bestehenden strukturellen Hemmnisse zu befördern. Vor diesem Hintergrund lädt die DGIV zu ihrem Bundeskongress ein, der sich dem Themenkomplex CORONA (Lessons learned/ Resilienz) widmet.

\section{Lehren aus der CORONA-Krise für die Integrierte Versorgung \\ - CORONA-Krise - Katalysator zur Überwindung der Sektorengrenzen \\ - Integrierte Versorgung - CORONA und danach \\ - Ethische Erkenntnisse aus der CORONA- Krise}

\section{Sektorenübergreifende Versorgung}

- Bewertung der Arbeitsergebnisse der Bund-Länder-Arbeitsgruppe sektorenübergreifende Versorgung

- Zu wenig Freiheit oder zu viele GruppenEgoismen? - Woran hakt es bei der Weiterentwicklung der integrierten Selektivversorgung?

- Wieviel „ambulant" können Krankenhäuser und wieviel "stationär" können Vertragsärzte leisten?
CORONA-Erfahrungen - die medizinische Perspektive

- CORONA-Erfahrungen aus erster Hand "Hotspot" Madrid

- CORONA-Erfahrungen aus der Hauptstadt Deutschlands - am Beispiel von Vivantes

- CORONA-Erfahrungen aus der Onkologie - wie sieht die Tumormedizin der Zukunft aus?

\section{Podiumsdiskussion „Wie sieht die} Roadmap nach CORONA aus?"

\section{Veranstalter}

DGIV e.V., Wartburgstraße 11, 10823 Berlin, Tel.: 030/44727080, Fax: 030/44729746,

E-Mail:info@dgiv.org

\section{Wissenschaftliche Leitung}

Prof. Dr. Stefan G. Spitzer, Dresden

Prof. Dr. Dr. Alfred Holzgreve, Berlin

Dr. Michael Meyer, Berlin

\section{Tagungsort}

Hotel Aquino, Tagungszentrum Katholische Akademie, Hannoversche Straße 5b, 10115 Berlin-Mitte

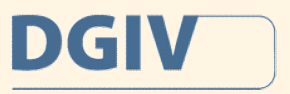

Deutsche Gesellschaft fiur Integrierte Versorgung im Gesundheitswesen e.

Quelle und weitere Infos: www.dgiv.org 